\title{
Men Are More Likely than Women to Slow in the Marathon
}

\author{
ROBERT O. DEANER ${ }^{1}$, RICKEY E. CARTER ${ }^{2}$, MICHAEL J. JOYNER ${ }^{3}$, and SANDRA K. HUNTER ${ }^{4}$ \\ ${ }^{1}$ Department of Psychology, Grand Valley State University, Allendale, MI; ${ }^{2}$ Department of Health Sciences Research, \\ Mayo Clinic, Rochester, MN; ${ }^{3}$ Department of Anesthesiology, Mayo Clinic, Rochester, MN; and ${ }^{4}$ Exercise Science Program, \\ Department of Physical Therapy, Marquette University, Milwaukee, WI
}

\begin{abstract}
DEANER, R. O., R. E. CARTER, M. J. JOYNER, and S. K. HUNTER. Men Are More Likely than Women to Slow in the Marathon. Med. Sci. Sports Exerc., Vol. 47, No. 3, pp. 607-616, 2015. Studies on nonelite distance runners suggest that men are more likely than women to slow their pace in a marathon. Purpose: This study determined the reliability of the sex difference in pacing across many marathons and after adjusting women's performances by $12 \%$ to address men's greater maximal oxygen uptake and also incorporating information on racing experience. Methods: Data were acquired from 14 US marathons in 2011 and encompassed 91,929 performances. For 2929 runners, we obtained experience data from a race-aggregating Web site. We operationalized pace maintenance as the percentage change in pace observed in the second half of the marathon relative to the first half. Pace maintenance was analyzed as a continuous variable and as two categorical variables, as follows: "maintain the pace," defined as slowing $<10 \%$, and "marked slowing," defined as slowing $\geq 30 \%$. Results: The mean change in pace was $15.6 \%$ and $11.7 \%$ for men and women, respectively $(P<0.0001)$. This sex difference was significant for all 14 marathons. The odds for women were $1.46(95 \%$ confidence interval, $1.41-1.50 ; P<0.0001)$ times higher than men to maintain the pace and 0.36 (95\% confidence interval, $0.34-0.38 ; P<0.0001)$ times that of men to exhibit marked slowing. Slower finishing times were associated with greater slowing, especially in men (interaction, $P<0.0001$ ). However, the sex difference in pacing occurred across age and finishing time groups. Making the $12 \%$ adjustment to women's performances lessened the magnitude of the sex difference in pacing but not its occurrence. Although greater experience was associated with less slowing, controlling for the experience variables did not eliminate the sex difference in pacing. Conclusions: The sex difference in pacing is robust. It may reflect sex differences in physiology, decision making, or both. Key Words: GENDER, DISTANCE RUNNING, RISK TAKING, ENDURANCE EXERCISE, EXPERIENCE, SUBSTRATE USE
\end{abstract}

$\mathrm{P}$ acing in endurance races has long been of interest to sports scientists, but research has been traditionally limited to elite or subelite athletes $(1,13,28,37)$. The recent availability of data from mass-participation events, however, has permitted pacing to be investigated in a broader range of athletes, including nonelite distance runners. Several notable patterns have emerged, including that men are more likely than women to slow their pace in the marathon $(42.195 \mathrm{~km})(26,35)$.

The sex difference in pacing could reflect physiological differences, such as men's greater susceptibility to muscle glycogen depletion $(7,31,34)$ or women's skeletal muscle showing less fatigability (19). An alternative, but compatible, possibility is that pacing differences may reflect decision

Address for correspondence: Sandra K. Hunter Ph.D., Exercise Science Program, Department of Physical Therapy, Marquette University, PO Box 1881, Milwaukee, WI 53201; E-mail: Sandra.Hunter@marquette.edu. Submitted for publication February 2014.

Accepted for publication June 2014.

0195-9131/15/4703-0607/0

MEDICINE \& SCIENCE IN SPORTS \& EXERCISE $_{\circledast}$ Copyright $\left({ }^{\circ} 2014\right.$ by the American College of Sports Medicine

DOI: $10.1249 /$ MSS.0000000000000432 making (2). In particular, men may be more likely to adopt a "risky" pace, where an individual begins the race with a fast early pace (relative to their ability), and this increases their likelihood of slowing later. If either of these possibilities proved correct, they would constitute a novel source of pacing variation, one that has not been considered in studies on elite or subelite athletes.

Here, we addressed the robustness of the sex difference in marathon pacing by extending previous studies in three ways. First, rather than focusing on one marathon, we evaluated a sample of 14 marathons, which encompasses data from over 91,000 performances. This allowed us to generalize our findings to a range of ages and a variety of races.

Second, we obtained information on racing experience for a subsample of participants $(>2900)$ and tested whether experience was related to pacing and whether it might contribute to the (possible) sex difference in pacing. A previous study reported that older runners showed more even marathon pacing and suggested that this reflected their greater racing experience (26). However, no previous study has directly tested whether race experience is associated with better pacing.

Third, we made a $12 \%$ adjustment or correction to women's performances to address the fact that they are roughly $10 \%-$ $12 \%$ slower than men's even when training and talents are 
similar $(8,20,21,24,32)$. This sex difference reflects men's greater maximal oxygen uptake $\left(\dot{\mathrm{V}}_{2 \max }\right)$, which is mediated by their larger hearts, greater hemoglobin concentration, less body fat, and greater muscle mass per unit of body weight $(8,20,21,24,32)$. Making this adjustment allows the alignment of relative performance between the sexes. This is potentially important because, at least in some populations (e.g., older runners), a larger proportion of men than women achieves relatively fast performances $(11,20,21)$ and relatively fast performances may, in turn, be associated with more even pacing (26). In other words, the previously reported sex difference in pacing $(26,35)$ might merely reflect that more men than women run relatively fast.

Although most of our analyses are based on making a $12 \%$ adjustment to women's performances, we also explored other adjustments. We used a $10 \%$ adjustment to address the commonly mentioned lower bound for the sex difference in $\dot{\mathrm{V}} \mathrm{O}_{2 \max }(8,19,22,26,34)$ and the $10 \%$ difference we observed in the mean finishing times for men and women in our sample. We used a $16 \%$ adjustment because the sex difference in endurance performance, although typically approximately $10 \%$ for world records and other leading performances, is often observed to be $14 \%-16 \%$ for nearelite performers $(11,20,32)$. Finally, to allow direct comparisons with previous studies $(2,28,37)$, we conducted analyses without any adjustment.

The purpose of this study, therefore, was to compare the pacing or pace maintenance (duration of the first half of the marathon relative to the second half) of men and women across different age groups and abilities (finishing times) in a large sample of marathons. A secondary purpose was to determine whether racing experience was related to pacing and whether it might contribute to the sex difference in pacing. A third purpose was to test whether adjusting women's performances to account for their lower $\dot{\mathrm{VO}}_{2 \max }$ would affect the sex difference in pacing.

\section{METHODS}

This study did not require formal approval by Grand Valley State's institutional review board (IRB) or the other IRB (Marquette University, Mayo Clinic). The Grand Valley State University IRB determined that the protocol (reference number 14-078-H) was exempt under federal category 45 CFR 46.101(b) (4) because all data were preexisting and public.

We began identifying potential marathons using marathon. guide.com, a Web site that attempts to maintain a comprehensive list. We initially considered all 75 marathons with more than 1000 finishers held in the United States in 2011. We discovered, however, that many races did not provide halfway times, and, among those races that did, data were often difficult to download efficiently. We therefore focused on the 14 races where halfway and full race data were presented with the active.com timing platform, which permitted efficient data acquisition.

Of the 96,774 individual times available, the final analysis set consisted of 91,929 (95\%) usable finishing times. Criteria for inclusion in the final data set were having timing data for the halfway mark and the full race in proper sequence (e.g., finishing time greater than half marathon time), a net time less than the gun time, and a slowing less than $400 \%$. The number of participants by marathon is presented in Table 1. The sample consisted of $41.5 \%$ women $(n=$ 38,130). The largest three races (Chicago, Disney, and Philadelphia) constituted $64.3 \%$ of the sample $(n=59,140)$, and each of the remaining 11 races comprised $1.2 \%-5.2 \%$ of the total participants.

To address racing experience, we obtained information from athlinks.com, a Web site that seeks to aggregate finishers' data from all races. Although some races included information as far back as the early 1980s, information was more comprehensive for races occurring since the early 2000s. We searched for each finisher's name and recorded

TABLE 1. Listing of marathon participants and summary data on percentage change in pace from the first half to the second half by sex.

\begin{tabular}{|c|c|c|c|c|c|c|c|c|c|c|c|c|c|}
\hline \multirow[b]{3}{*}{ Marathon } & \multicolumn{4}{|c|}{ Total Sample } & \multicolumn{4}{|c|}{ Women } & \multicolumn{4}{|c|}{ Men } & \multirow[b]{3}{*}{$P$ Value $^{c}$} \\
\hline & \multicolumn{2}{|c|}{ Participants } & \multicolumn{2}{|c|}{$\begin{array}{c}\text { Percentage } \\
\text { Change in Pace } \\
(\%)\end{array}$} & \multicolumn{2}{|c|}{ Participants } & \multicolumn{2}{|c|}{$\begin{array}{c}\text { Percentage } \\
\text { Change in Pace } \\
(\%)\end{array}$} & \multicolumn{2}{|c|}{ Participants } & \multicolumn{2}{|c|}{$\begin{array}{c}\text { Percentage } \\
\text { Change in Pace } \\
(\%)\end{array}$} & \\
\hline & Count & Pct. $^{a}$ & Mean & SD & Count & Pct. $^{b}$ & Mean & SD & Count & Pct. $^{b}$ & Mean & $\overline{S D}$ & \\
\hline Air Force & 3199 & 3.5 & 19.2 & 14.9 & 1042 & 32.6 & 16.9 & 12.1 & 2157 & 67.4 & 20.3 & 16.0 & $<0.0001$ \\
\hline Atlanta & 1109 & 1.2 & 13.8 & 12.1 & 422 & 38.1 & 11.5 & 9.6 & 687 & 62.0 & 15.2 & 13.2 & 0.0006 \\
\hline Austin & 4792 & 5.2 & 15.2 & 13.0 & 1941 & 40.5 & 12.1 & 10.2 & 2851 & 59.5 & 17.3 & 14.2 & 0.0003 \\
\hline Baltimore & 3188 & 3.5 & 17.7 & 15.1 & 1189 & 37.3 & 14.4 & 12.4 & 1999 & 62.7 & 19.7 & 16.2 & $<0.0001$ \\
\hline Chicago & 35,571 & 38.7 & 16.0 & 13.1 & 15,412 & 43.3 & 13.5 & 10.9 & 20,159 & 56.7 & 17.9 & 14.2 & $<0.0001$ \\
\hline Cleveland & 2598 & 2.8 & 10.3 & 11.0 & 994 & 38.3 & 9.6 & 10.0 & 1604 & 61.7 & 10.7 & 11.5 & 0.0317 \\
\hline Dallas & 4473 & 4.9 & 10.5 & 11.4 & 1702 & 38.1 & 8.6 & 9.4 & 2771 & 62.0 & 11.6 & 12.3 & $<0.0001$ \\
\hline Disney & 13,431 & 14.6 & 9.4 & 11.7 & 6225 & 46.4 & 8.1 & 10.1 & 7206 & 53.7 & 10.5 & 12.9 & $<0.0001$ \\
\hline Georgia & 2201 & 2.4 & 13.7 & 12.0 & 788 & 35.8 & 10.9 & 9.1 & 1413 & 64.2 & 15.2 & 13.1 & $<0.0001$ \\
\hline Grand Rapids & 1581 & 1.7 & 11.3 & 13.5 & 697 & 44.1 & 9.5 & 10.1 & 884 & 55.9 & 12.7 & 15.6 & 0.0011 \\
\hline Miami & 3498 & 3.8 & 12.3 & 12.5 & 1245 & 35.6 & 10.6 & 10.7 & 2253 & 64.4 & 13.2 & 13.3 & $<0.0001$ \\
\hline Philadelphia & 10,138 & 11.0 & 13.1 & 12.4 & 4209 & 41.5 & 11.1 & 10.6 & 5929 & 58.5 & 14.6 & 13.3 & $<0.0001$ \\
\hline Pittsburgh & 4255 & 4.6 & 10.8 & 11.0 & 1624 & 38.2 & 9.0 & 9.0 & 2631 & 61.8 & 11.9 & 12.0 & $<0.0001$ \\
\hline St. Louis & 1895 & 2.1 & 21.6 & 13.2 & 640 & 33.8 & 18.6 & 11.8 & 1255 & 66.2 & 23.1 & 13.6 & $<0.0001$ \\
\hline Total & 91,929 & & 14.0 & 13.0 & 38,130 & 41.5 & 11.7 & 10.9 & 53,799 & 58.5 & 15.6 & 14.2 & \\
\hline
\end{tabular}

${ }^{a}$ Percentage of participants in the total sample from the individual race.

${ }^{b}$ Percentage of women and men participants within race.

${ }^{c} P$ value from post hoc comparison of percentage change between sexes for each marathon. 
their total number of races, total number of marathons, personal bests for the $5 \mathrm{~km}$ and marathon, and earliest year with a recorded race.

Searches for some finishers' names yielded race data from more than one individual (e.g., several people named Jane Jones). In some cases, we were able to determine which races were completed by the marathon finisher of interest because of unique identifiers (e.g., age, hometown). In addition, some runners "claimed" a series of races that they had finished, and these performances thus became identified with a unique member profile. In cases with ambiguities and/or no member profile, we excluded the marathoner of interest. Obtaining experience and ability data in this manner was highly time consuming, so we only investigated a sample of finishers. In particular, we generated a randomized list of approximately 6000 marathoners from the full sample of 91,929 performances; we were able to obtain experience data for 2929 of these.

Three research assistants participated in the acquisition of the experience data, and we investigated the reliability of their work by assigning two of them to independently code 210 marathon finishers who had been previously assigned to other coders. Intercoder reliability for identifying finishers was modest (Cohen $\kappa=0.51$ ); in 85 cases, both coders identified the marathon finisher in athlinks.com; in 75 cases, neither identified the finisher; and in 52 cases, one coder but not the other identified the finisher. In cases where both coders identified the finisher, their agreement was good, as follows: total previous races, $r(83)=0.97, P<0.0001$; previous marathons, $r(83)=0.97, P<0.0001$; fastest previous marathon, $r(83)=0.96, P<0.0001$; fastest $5 \mathrm{~km}, r(40)=$ $0.99, P<0.0001$; and earliest year with a recorded race, $r(83)=0.91, P<0.0001$.

Statistical analysis. The overarching goal of the analysis was to test for sex differences in patterns of pace maintenance over the marathon while adjusting for men's generally faster performances. Furthermore, we sought to control for the potential confounding effects of age and running ability through stratification. Total finishing time, first half time, and second half time were determined from chip times (i.e., when each individual crossed the starting line). The finishing times for men were categorized into $<3 \mathrm{~h} 00 \mathrm{~min}$ up to $5 \mathrm{~h}$ or more in 30-min increments. Women's categories were shifted up by $12 \%$ to account for the sex difference in $\dot{\mathrm{VO}}_{2 \max }$. For example, the $<3 \mathrm{~h} 00 \mathrm{~min}$ boundary for men would equate to a $3 \mathrm{~h} 22 \mathrm{~min}$ boundary for women. For analyses using pacing as a continuous variable, the chip times of women were divided by 1.12 to introduce the $12 \%$ adjustment.

Pace maintenance was operationalized by calculating the percentage change in the pace observed in the second half of the marathon relative to the first half pace $(\%$ change $=$ (second half time - first half time)/first half time). This percentage change was used as a continuous variable and for two categorizations. For the first categorization, percentage changes less than $10 \%$ were considered "maintaining the pace." For example, a runner who completed the first half of the marathon in $2 \mathrm{~h} 0 \mathrm{~min}$ and completed the second half in $2 \mathrm{~h} 11 \mathrm{~min}$ or faster maintained the pace; those who took $2 \mathrm{~h}$ 12 min or slower failed to maintain the pace. For the second categorization, percentage changes greater than $30 \%$ were considered as "marked slowing." This classification was created to represent a transition from running in the first half to considerable walking during the second half of the race. For example, a runner who completed the first half of the marathon in $2 \mathrm{~h} 0 \mathrm{~min}$ and the second half in $2 \mathrm{~h} 36 \mathrm{~min}$ or slower exhibited marked slowing. Mantel-Haenszel techniques for stratified data were used to summarize the odds for maintaining, or not, the pace during the race when controlled for age, first half pace, and race. In instances where the odds ratio (OR) was not defined because of a zero cell, the empirical logit was used for computation. This estimator for the OR added 0.5 to each of the cell before the calculation of the stratum-specific OR and the overall pooled estimate.

These categorizations enabled ease of interpretation and graphical display. To ensure statistical efficacy, regression analyses were also used to test for coincidence of regression lines for men versus women using the continuous percentage change in pace by finishing time. In addition, multiple linear regression techniques were used to explore the relation of experience variables with the percentage change in pace.

TABLE 2. Percentage slowing by sex and finishing time group. Finishing time groups for women have been adjusted by $12 \%$ to account for $\dot{V} \mathrm{O}_{2 \mathrm{max}}$ differences.

\begin{tabular}{|c|c|c|c|c|c|c|c|c|}
\hline \multirow[b]{2}{*}{ Sex } & \multirow[b]{2}{*}{ Finishing Time } & \multirow[b]{2}{*}{$n$} & \multirow[b]{2}{*}{ Mean (\%) } & \multirow[b]{2}{*}{ SD (\%) } & \multicolumn{2}{|c|}{$\begin{array}{l}\text { Maintained the Pace } \\
(<\mathbf{1 0} \% \text { Slowing })\end{array}$} & \multicolumn{2}{|c|}{$\begin{array}{l}\text { Marked Slowing } \\
(\geq 30 \% \text { Slowing) }\end{array}$} \\
\hline & & & & & $n$ & Pct. & $n$ & Pct. \\
\hline \multirow[t]{6}{*}{ Women } & $<3$ h 22 min & 677 & 5.0 & 6.0 & 575 & 84.9 & 2 & 0.3 \\
\hline & $<3$ h $55 \mathrm{~min}$ & 4041 & 5.7 & 6.5 & 3184 & 78.8 & 13 & 0.3 \\
\hline & $<4$ h 29 min & 8504 & 8.7 & 8.4 & 5192 & 61.1 & 125 & 1.5 \\
\hline & $<5$ h 02 min & 9824 & 11.3 & 10.1 & 4785 & 48.7 & 436 & 4.4 \\
\hline & $<5$ h $36 \min$ & 6912 & 14.6 & 11.5 & 2506 & 36.3 & 585 & 8.5 \\
\hline & $5 \mathrm{~h} 36 \mathrm{~min}$ or more & 8172 & 16.5 & 12.5 & 2461 & 30.1 & 930 & 11.4 \\
\hline \multirow[t]{6}{*}{ Men } & $<3$ h $00 \mathrm{~min}$ & 1355 & 5.6 & 6.6 & 1096 & 80.9 & 12 & 0.9 \\
\hline & $<3$ h 30 min & 5534 & 7.6 & 8.1 & 3809 & 68.8 & 85 & 1.5 \\
\hline & $<4$ h $00 \mathrm{~min}$ & 11,439 & 10.3 & 10.4 & 6386 & 55.8 & 529 & 4.6 \\
\hline & $<4$ h $30 \mathrm{~min}$ & 11,732 & 15.0 & 12.4 & 4474 & 38.1 & 1283 & 10.9 \\
\hline & $<5$ h $00 \mathrm{~min}$ & 10,413 & 18.6 & 14.3 & 3109 & 29.9 & 2021 & 19.4 \\
\hline & $5 \mathrm{~h} 00 \mathrm{~min}$ or more & 13,326 & 22.7 & 16.5 & 2727 & 20.5 & 3586 & 26.9 \\
\hline
\end{tabular}

Finishing time groups are listed as non-mutually exclusive categories for brevity in presentation. For example, " $<3 \mathrm{~h} 55$ min" corresponds to the stratum of finishers having finishing times of 3 h 22 min or greater but less than 3 h 55 min. 


\section{A Maintaining the Pace}

\section{Women}

$\mathrm{n} / \mathrm{N}$

Pace Group

$\begin{array}{lrr}<3: 00 \mathrm{M},<3: 22 \mathrm{~F} & 575 / 677 & 1096 / 1355 \\ <3: 30 \mathrm{M},<3: 55 \mathrm{~F} & 3184 / 4041 & 3809 / 5534 \\ <4: 00 \mathrm{M},<4: 29 \mathrm{~F} & 5192 / 8504 & 6386 / 11439 \\ <4: 30 \mathrm{M},<5: 02 \mathrm{~F} & 4785 / 9824 & 4474 / 11732 \\ <5: 00 \mathrm{M},<5: 36 \mathrm{~F} & 2506 / 6912 & 3109 / 10413 \\ & & \\ 5: 00+\mathrm{M}, 5: 36+\mathrm{F} & 2461 / 8172 & 2727 / 13326\end{array}$

Total

$18703 / 38130$

21601/53799
Odds Ratio $(95 \% \mathrm{Cl})$

Odds Ratio $(95 \% \mathrm{Cl})$

$1.33(1.04$ to 1.71$)$

$1.68(1.53$ to 1.85$)$

$1.24(1.17$ to 1.31$)$

1.54 (1.46 to 1.63$)$

1.34 (1.25 to 1.43$)$

$1.67(1.57$ to 1.78$)$

$1.46(1.41$ to 1.50$)$

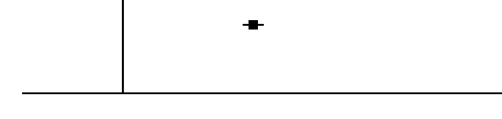

$0.75 \quad 1$

3

Favors Men Favors Women

\section{B Marked Slowing}

Women

$\mathrm{n} / \mathrm{N}$

Pace Group

$<4: 30 \mathrm{M},<5: 02 \mathrm{~F}$

$\begin{array}{rr}<3: 00 \mathrm{M},<3: 22 \mathrm{~F} & 2 / 677 \\ <3: 30 \mathrm{M},<3: 55 \mathrm{~F} & 13 / 4041\end{array}$

$<4: 00 \mathrm{M},<4: 29 \mathrm{~F} \quad 125 / 8504$

$<5: 00 \mathrm{M},<5: 36 \mathrm{~F}$

$5: 00+M, 5: 36+F$

$436 / 9824$

$585 / 6912$

$930 / 8172$

$2091 / 38130 \quad 7516 / 53799$

$$
12 / 1355
$$

$85 / 5534$

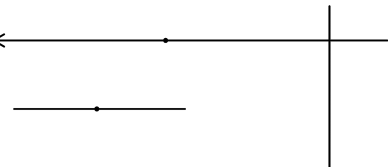

$529 / 11439$

$1283 / 11732$

$2021 / 10413$

$3586 / 13326$

Total

$$
2091 / 38130
$$

Odds Ratio $(95 \% \mathrm{Cl})$

$0.33(0.07$ to 1.49$)$

$0.21(0.12$ to 0.37$)$

$0.31(0.25$ to 0.37$)$

$0.38(0.34$ to 0.42$)$

$0.38(0.35$ to 0.42$)$

0.35 (0.32 to 0.38$)$

0.36 (0.34 to 0.38$)$

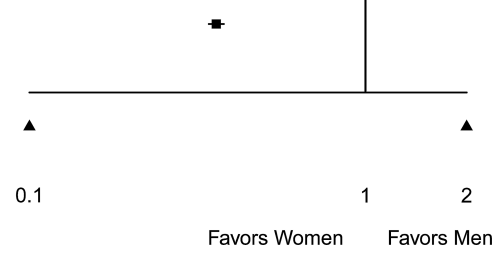

FIGURE 1-OR of maintaining pace and marked slowing. Forest plot for the odds of maintaining the pace ( $<10 \%$ change in pace) (A) and marked slowing ( $\geq 30 \%$ change in pace) (B) for women relative to men. The error bars are $95 \%$ CI. The common estimate is the pooled OR as estimated by the Mantel-Haenszel estimator. "Favors women" indicates that women were less likely to slow than men. Conversely, "favors men" means that men were less likely to slow than women. 
All statistical analyses were conducted using the SAS system version 9.3 (Cary, NC). Two-sided $P$ values less than 0.05 were taken as statistically significant.

\section{RESULTS}

A total of 91,929 runners (41.5\% women) were included in this analysis. The mean \pm SD ages for men and women were $38.9 \pm 10.8$ and $35.5 \pm 9.7 \mathrm{yr}$. The mean finishing times for men and women were $4 \mathrm{~h} 28 \mathrm{~min} \pm 53 \mathrm{~min}$ and $4 \mathrm{~h} 54 \mathrm{~min} \pm$ 52 min, a difference of $10 \%$. The mean \pm SD changes in pace were $15.6 \% \pm 14.2 \%$ and $11.7 \% \pm 10.9 \%$ for men and women, respectively $(P<0.0001)$. Although there was variability across marathons, women consistently showed less change in pace $(P<0.05$ for all 14 marathons) (Table 1$)$.

Pacing and finishing time. Table 2 reports the distribution of percentage change in pace (duration of first half of the marathon relative to the second half) by finishing time group separately for men and women. The finishing time groups for women have been adjusted by $12 \%$, so they are comparable with the performances of men. The percentage change in pace varied across finishing time group for each sex $(P<0.0001$ for both sexes). Table 2 shows that slower groups had lower percentages of participants who maintained the pace $(<10 \%$ slowing $)$ and more participants $(\%)$ who exhibited marked slowing ( $\geq 30 \%$ slowing). This pattern of greater slowing with greater finishing times was more pronounced in men than in women (see following section).
We calculated the common (pooled) OR for maintaining the pace (Fig. 1A) and marked slowing (Fig. 1B) for women relative to men across the finishing time groups. Overall, women were 1.46 times (95\% confidence interval (CI), $1.41-1.50 ; P<0.0001)$ more likely to maintain the pace $(<10 \%$ slowing) compared with men. There were, however, differences among the finishing time groups $(P<0.0001$, indicated by a Breslow-Day test for homogeneity). The largest sex differences occurred in the subelite category (3:00-3:30 for men and 3:22-3:55 for women; OR, 1.68; 95\% CI, 1.53-1.85) and those with the slowest finishing times (5:00+ for men and 5:36+ for women; OR, 1.67; 95\% CI, 1.57-1.78).

Overall, $5 \%$ of women and $14 \%$ of men experienced marked slowing ( $\geq 30 \%$ slowing), which resulted in women having $64 \%$ lower odds for marked slowing than men (OR, 0.36 ; 95\% CI, 0.34-0.38) (Table 2 and Fig. 1B). This pattern of women showing less odds of marked slowing did not differ significantly across finishing time groups (BreslowDay test, $P=0.11)$. However, there were only 14 participants ( 12 women and two men) in the elite group ( $<3: 00$ men; $3: 22$ women), so that the precision of estimated OR was poor (wide CI).

Pacing and age. Figures 2 and 3 display the OR of maintaining the pace and marked slowing for women relative to men, with stratification for finishing time group and age group. The overall (pooled) estimate of the common OR for maintaining the pace with adjustment for finishing time and

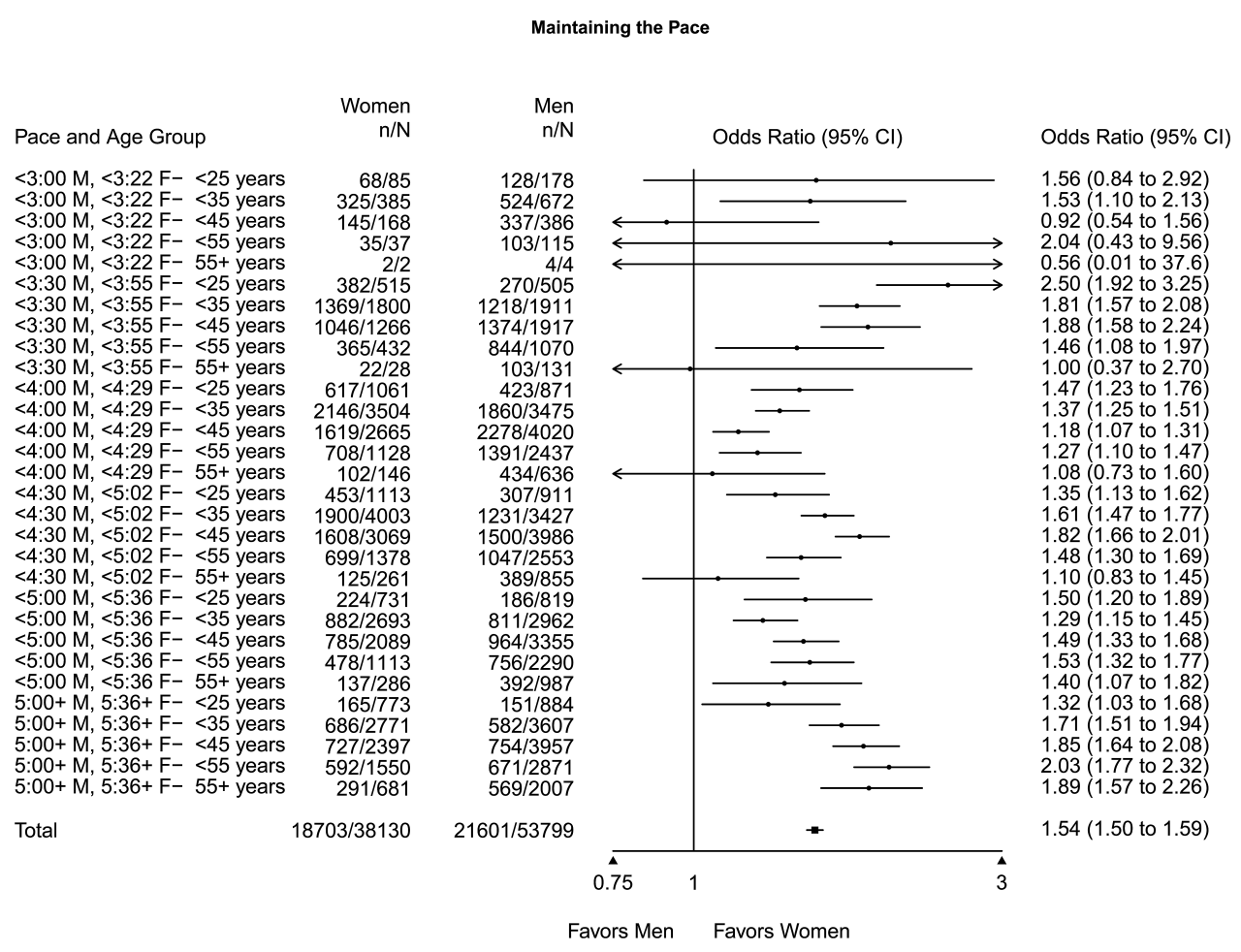

FIGURE 2-OR of maintaining pace. Forest plot for the odds of maintaining the pace ( $<\mathbf{1 0 \%}$ change in pace) for women relative to men with stratification for finishing time group and age group. The error bars are $95 \% \mathrm{CI}$. The common estimate is the pooled OR as estimated by the MantelHaenszel estimator. The empirical logit estimator for the OR was used when at least one sex category had no (or all) event (e.g., all men and women in the $<3$-h marathon finishing time group). 


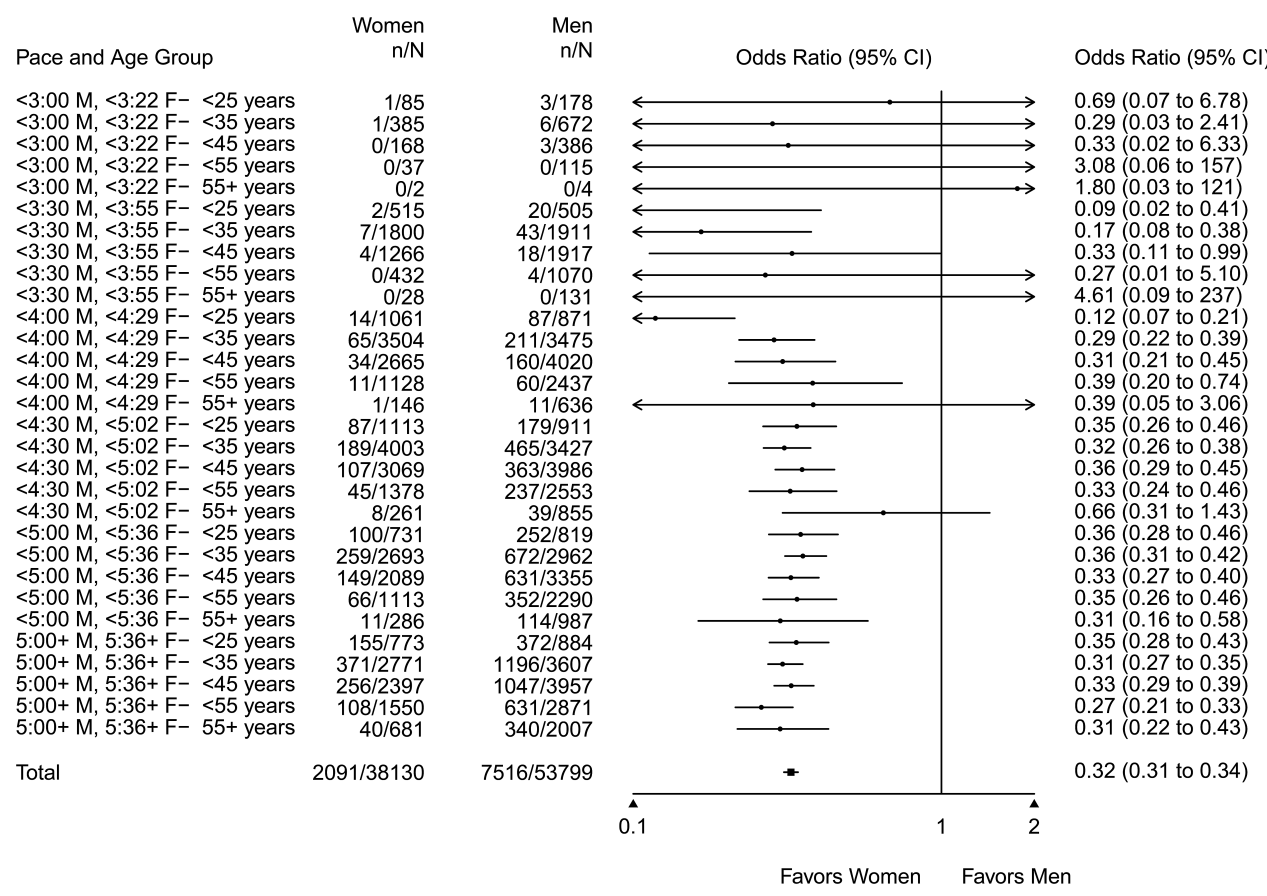

FIGURE 3-OR of marked slowing. Forest plot for the odds of marked slowing ( $\geq \mathbf{3 0} \%$ change in pace) for women relative to men with stratification for finishing time group and age group. The error bars are $95 \%$ CI. The common estimate is the pooled OR as estimated by the Mantel-Haenszel estimator. The empirical logit estimator for the OR was used when at least one sex category had no (or all) event (e.g., all men and women in the $<3$-h marathon finishing time group).

age was $1.54(95 \% \mathrm{CI}, 1.50-1.59 ; P<0.001)$ for women relative to men, although there was significant variation across groups (Breslow-Day test, $P<0.001$ ) (Fig. 2). For marked slowing, the common OR with adjustment for finishing time and age was $0.32(95 \% \mathrm{CI}, 0.31-0.34 ; P<$ 0.001 ) for women relative to men. Similar to the results presented in Figure 1B, patterns were similar across finishing time and age groups (Breslow-Day test, $P=0.19$ ) (Fig. 3).

Change in pace as a continuous measure. To assess the sensitivity of categorizing finishing time and percentage slowing, we conducted regression analyses. Men and women slowed differentially $\left(F_{2,91925}=993, P<\right.$ 0.0001 ), so that the sex difference widened among slower runners (adjusted for women relative to men by 12\%). For instance, percentage slowing for a $3 \mathrm{~h} 00 \mathrm{~min}$ male finisher and $3 \mathrm{~h} 26 \mathrm{~min}$ female finisher (compared because of the $12 \%$ adjustment for finishing time) were $6.9 \%$ and $5.5 \%$, respectively ( $25 \%$ difference). However, for a 5 h $00 \mathrm{~min}$ male finisher and a $5 \mathrm{~h} 36 \mathrm{~min}$ female finisher ( $12 \%$ adjusted), slowing widened to $18.8 \%$ and $14.5 \%$, respectively $(30 \%$ difference). This regression model explained $20 \%\left(R^{2}=\right.$ 0.197 ) of the variation in percentage change in pace, but the relations were not strictly linear.

To examine the nonlinear relation more fully, Figure 4A illustrates a locally weighted (LOESS smoother) plot relating finishing times with percentage change in pace for the entire sample. A scatter plot consisting of a random sample of 5000 finishers has been superimposed onto the figure to add context to these regression fits. Percentage slowing increases more rapidly in men than in women as finishing time increases.

Sensitivity analysis for the $12 \%$ adjustment. We next investigated whether our results were sensitive to the $12 \%$ adjustment we made to women's performances. Figure 4B displays locally weighted plots relating finishing times to change in pace for the entire sample using no adjustment, $10 \%$ adjustment, and $16 \%$ adjustment. No adjustment produced a larger estimate of the sex difference in pacing than that when using adjustment $(10 \%$ or $16 \%)$. Furthermore, the sex difference in pacing was smaller with the $16 \%$ adjustment than with the $12 \%$ adjustment used in our earlier analyses (Fig. 4A). Nonetheless, even with $16 \%$ adjustment, the sex difference in pacing remained apparent in all analyses as noncoincident regression lines (i.e., regression lines of women generally indicating less slowing for any given finishing time).

Race experience. Finally, we explored the effect of experience on pacing in a subgroup of 2929 runners. These runners had a mean finishing time of $4 \mathrm{~h} 36 \mathrm{~min}$ and included $42.8 \%$ female participants. The overall mean \pm SD percentage change in pace was $15.0 \% \pm 13.5 \%$. As with the entire sample, men's percentage change in pace was greater than the women's $(16.8 \%$ vs $12.5 \% ; P<0.0001)$. Similar to the entire sample, $12.4 \%$ of the racers showed marked slowing, with men showing higher frequency than women $(16.7 \%$ vs $6.6 \%$ respectively, $P<0.0001$ ). 


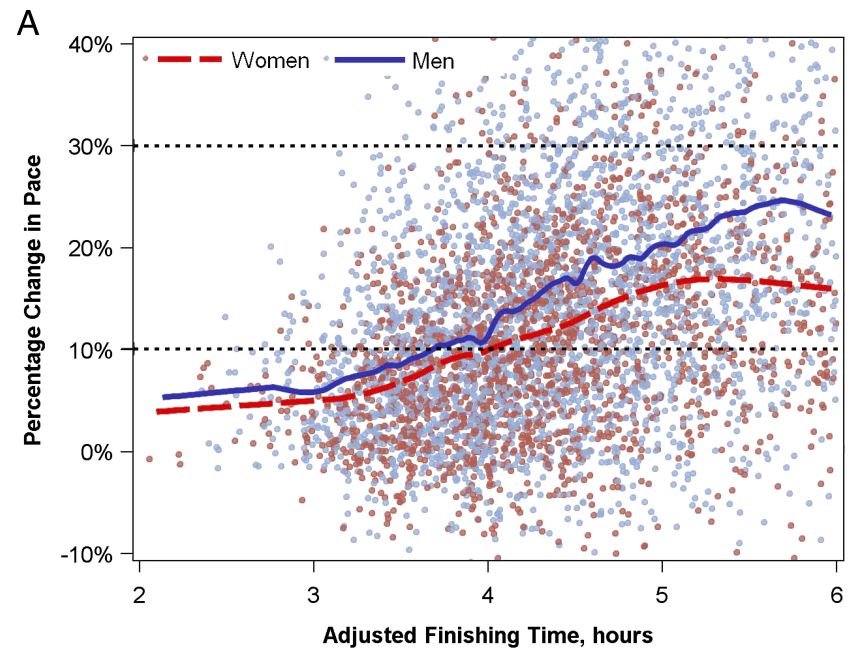

B

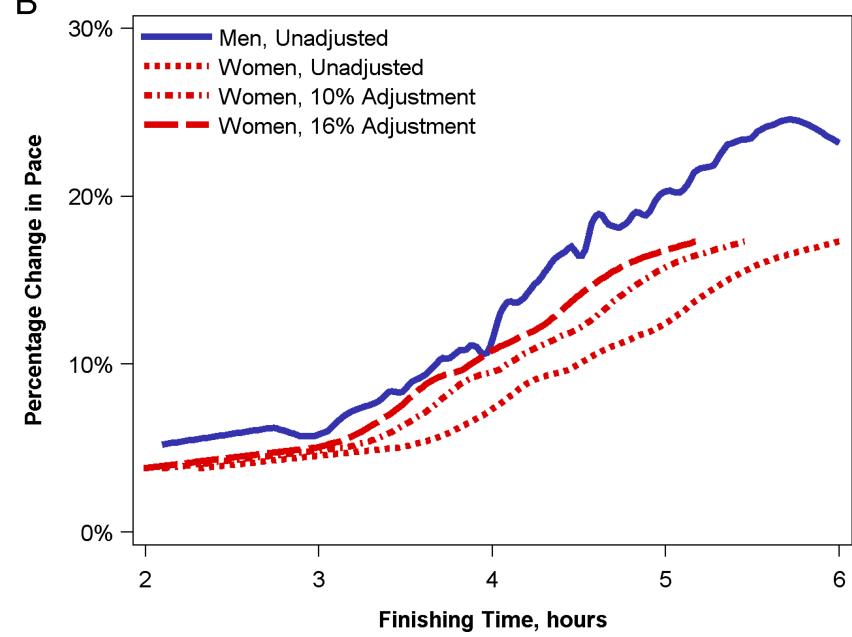

FIGURE 4-Percentage change in pace as a function of finishing time for men and women. A. LOESS-smoothed curves modeling the relation of percentage change in pace as a function of finishing times and for men and women who finished under $6 \mathrm{~h}(n=84,277)$. The points plotted are a random sample of $\mathbf{5 0 0 0}$ finishers to provide context to the LOESS-fitted lines. Women's finishing times have been divided by $\mathbf{1 . 1 2}$ to account for $\dot{\mathrm{V}} \mathrm{O}_{2 \max }$ differences. B. LOESS-smoothed curves modeling the relation of percentage change in pace as a function of finishing times under $6 \mathrm{~h}(n=84,277)$ that are unadjusted and adjusted for physiological sex differences. "Women, 10\% adjustment" indicates that women's finishing times were divided by 1.10 ; “Women, $16 \%$ adjustment" indicates that women's finishing times were divided by 1.16 .

The next step was assessing which experience variables were significantly related to uneven pacing. We found greater slowing among those individuals with their earliest recorded race after 2008 ( $\leq 3 \mathrm{yr}$ of experience) $(\rho=0.08, P<$ $0.001)$, those with fewer previous marathons $(\rho=-0.08$, $P<0.001)$, and those with fewer total races at all distances $(\rho=-0.07, P<0.001)$. There was also greater pace slowing among those whose fastest previous marathons were slower $(\rho=0.19, P<0.001$, in 1856 participants that ran more than one marathon).

Consistent with the findings with the entire data set, the relation of the $12 \%$ adjusted finish time and percentage slowing differed between men and women (test for coincidence, $\left.F_{2,2925}=38.6 ; P<0.001\right)$. This model explained $20 \%$ of the variation in changes in pacing $\left(R^{2}=0.197\right)$. With the addition of participant age and the experience variables of total number of previous marathons and recent introduction to marathons ( $\leq 3 \mathrm{yr}$ of experience), $r^{2}$ increased to $20.8 \%$ $\left(n=2910, R^{2}=0.208\right)$. There was no evidence that the experience variables affected pacing differently in men and women $\left(F_{2,2901}=2.06, P=0.13\right)$. In a final model, consisting of the $12 \%$ adjusted finishing time, age, total marathons run, recent introduction to the marathon, and sex (the main effects of sex and its interaction with finishing time), the coefficients of partial determinations were as follows: $17.7 \%, 0.1 \%,<0.1 \%,<0.1 \%$, and $2.9 \%$, respectively. Thus, finishing time and the differences between men and women explained the largest percentages of the variation in the change in pace. The estimated percentage point differences in pace change, men relative to women, for relative finish times of $2 \mathrm{~h} 30 \mathrm{~min}$ (i.e., female finish time of $2 \mathrm{~h}$ $48 \mathrm{~min}), 3 \mathrm{~h} 00 \mathrm{~min}$, and $5 \mathrm{~h} 00 \mathrm{~min}$ were $1.6(P=0.15)$, $2.2(P=0.001)$, and $4.7(P<0.001)$ percentage points after adjusting for these experience variables.

\section{DISCUSSION}

This study shows that the sex difference in marathon pacing among nonelite runners is robust in several respects. First, the sex difference was significant in all 14 marathons in our sample. These marathons differed considerably in their size and extent of slowing (Table 1), indicating that the sex difference occurs generally. Second, we showed that, although age (i.e., being an older runner) and faster performances are associated with more even pacing (26), the sex difference generally persists across ages and abilities. There was evidence, however, for an interaction, with a less pronounced sex difference among faster runners. Third, we demonstrated that adjusting women's performances by $12 \%$ - to address men's greater $\dot{\mathrm{V}} \mathrm{O}_{2 \max }$ - does not eliminate the sex difference in pacing. Fourth, we found that, although inexperience is associated with greater pace slowing, controlling for experience does not eliminate the sex difference. Finally, our study is notable in its exploration of pace slowing as both a continuous and a categorical variable. Thus, rather than merely stating that men slowed more than women on average, we showed that, after controlling for age and ability and making the $12 \%$ adjustment, women had approximately one-third the odds as men to experience marked slowing (running the second half of the race more than $30 \%$ slower than the first half).

Besides demonstrating the robustness of the sex difference in pacing, our study yielded other notable results. One finding is that, making a $12 \%$ adjustment to women's performances did affect the magnitude of the sex difference in pacing. In particular, the sex difference was larger without an adjustment, indicating that previous studies that did not make an adjustment may have overestimated the sex difference in pacing $(26,35)$. Nonetheless, even with the $12 \%$ 
adjustment, evidence for the sex difference in pacing remained reliable. We also showed that although the estimated sex difference in pacing is generally insensitive to the exact adjustment used (between 10\% and 16\%), the sex difference in pacing decreases as the adjustment increases.

Our study also provided the first results directly bearing on the hypothesis that experience is related to pacing in the marathon (26). We showed that four experience variables were significantly associated with pacing, namely, years of experience, previous marathons, previous races at all distances, and fastest previous marathon. Thus, more experienced runners paced more evenly and inexperience was associated with greater pace slowing. Moreover, at least two of the experience variables (total marathon run and recent introduction to the marathon) explained variation in pacing independent of the effects of age, finishing time, and sex. The amount of variation explained by the experience variables, however, was modest $(>0.1 \%$ for each) compared with sex $(2.9 \%)$ and finishing time (17.7\%). Consequently, controlling for experience does not eliminate the sex difference.

What causes the sex difference in pacing? Various physiological factors might contribute to the sex difference in marathon pacing, although we did not measure these. For example, men are more susceptible to muscle glycogen depletion, which can contribute to greater fatigability and "hitting the wall" or dramatic marathon slowing $(4,9,30,33)$. Men may be more susceptible to slowing because, during endurance exercise, women generally have a lower RER, indicating that they use proportionately more fat and less $\mathrm{CHO}$ at a given intensity of exercise $(7,25,34,36)$. In general, women possess larger proportional areas of Type I muscle fibers, which are more resistant to fatigue especially for long-duration exercise (19). Supporting this hypothesis are studies finding that men are more likely than women to report dramatic slowing or hitting the wall $(4,33)$.

Another possibility is that men might be more susceptible to hyperthermia (35), which is believed to be another frequent contributor to marathon slowing $(16,27)$. Supporting this is the finding that the sex difference in marathon pacing in nonelites was larger in the 2007 Chicago marathon, which was hot $\left(27^{\circ} \mathrm{C}\right)$, than that in the 2009 Chicago marathon, which was cool $\left(3^{\circ} \mathrm{C}\right)(35)$. Challenging this hypothesis, however, are studies indicating that men typically enjoy advantages in thermoregulation (14).

Decision making could also contribute to the sex difference in marathon pacing. Specifically, if men are more likely to adopt a "risky" or competitive pace relative to their ability, this could increase their likelihood of slowing later. This is because a fast pace has numerous physiological consequences, including increasing the risk of hyperthermia and glycogen depletion $(3,9,13,37)$. Evidence for a sex difference in pacing decisions comes from a study showing that marathon performances among nonelites tend to cluster at round numbers (e.g., just under $4 \mathrm{~h}$ ) because of some runners speeding up in the final $2.2 \mathrm{~km}$ of the race; this pattern was substantially more pronounced among men than among women (2). There is also evidence that male distance runners are more likely to adopt a competitive rather than a recreational orientation towards the sport, as follows: 1) more male than female runners report that competition motivates them to run $(6,22,29), 2)$ roughly three times as many men as women run fast relative to sex-specific world-class standards, and this may reflect that that more men engage in the training necessary for faster performances (11), and 3) when men and women have the option of entering a single-sex competitive road race or a single-sex noncompetitive road race, men are more likely than women to select the competitive race (15). In addition, there is much evidence for sex differences in risk taking in other contexts, including health, recreation, and finance $(5,10,18)$.

Does the sex difference narrow among faster runners? Another interesting question is whether the sex difference in pacing is less among faster runners. Addressing this question is important because it could provide clues about the cause(s) of the sex difference. Within financial domains, for example, studies report that the sex difference in risk taking substantially weakens or disappears when financial knowledge is controlled $(12,17,23)$.

In the present study, despite the large overall sample size, there were very little data on genuinely elite or even near-elite runners. Moreover, faster runners, both men and women, generally maintain even paces (Table 2 and Fig. 4A). Notwithstanding these considerations, we did have some relevant results, although they provide an ambiguous answer to the question of whether the sex difference in pacing narrows among faster runners. On the one hand, the sex difference in pacing occurred among all finishing time groups (Table 2 and Fig. 1), and among runners in our two fastest groups (men running faster than 3:00 or 3:30; women running faster than $3: 22$ or 3:55), the magnitude of the sex difference based on categorical variables (i.e., OR) was similar to the sex difference across all finishing time groups (Table 2 and Fig. 1). On the other hand, when using continuous measures, the significant interaction of sex with adjusted finishing time indicates that the sex effect was significantly less pronounced in faster runners (Fig. 4A). Specifically, we estimated that among faster runners ( $3 \mathrm{~h} 00 \mathrm{~min}$ male finisher and corresponding $3 \mathrm{~h} 26 \mathrm{~min}$ female finisher), men slowed, on average, $25 \%$ more than women, whereas among slower runners $(5 \mathrm{~h} 00 \mathrm{~min}$ male finisher and corresponding 5 h 36 min female finisher), men slowed, on average, $30 \%$ more than women.

We note that there have been several studies on pacing among elite men and women in other endurance sports. These typically indicate no pacing differences (28) or else subtle differences that are difficult to interpret because of sex-specific aspects of the competition, such as the depth of competitors (37).

Are women more effective pacers? There is consensus that even (or nearly even) pacing is best for optimizing performance in races that take several hours to complete, such as marathons (1). Furthermore, dramatic pace slowing in a marathon is associated with considerable discomfort $(4,33)$. Our results therefore imply that women are generally more effective than men in their marathon pacing. Nonetheless, this 
conclusion should be considered provisional because an evenly paced race does not automatically indicate a well-paced one. This is because an individual may be overly conservative given their training and ability. Similarly, although dramatic slowing in a race almost certainly indicates a poor outcome for that race, a risky initial pace may be desirable in a broader context. For instance, if a runner's goal is to achieve at least one exceptional performance during the year (e.g., setting a personal record, winning their age group), he/she may have the best chance of achieving it if he/she often begin his/her races with a fast risky pace. Thus, a definitive answer to the question of whether particular groups or individuals are better pacers requires comprehensive assessment of goals.

Insights from nonelites. Much has been gained from studying the pacing of elite and subelite endurance athletes (1), and investigating the pacing of nonelite or recreational athletes may provide further insights. For example, besides sex difference, our study confirms that older runners and runners with faster initial pace tend to pace more evenly (26) and, to our knowledge, none of these effects were predicted by studies on elite pacing. Moreover, the variation in pacing among nonelites shown in our study (Fig. 4A) seems far greater than that among elites (13). This is probably due to the tremendous diversity of nonelites' goals, training, and abilities $(2,11)$. We suggest that future studies of pacing in nonelites assess these factors and others (e.g., coaching, fluid and $\mathrm{CHO}$ ingestion, use of pace groups, and targeted pace) to illuminate the interactions among physiological and psychological factors in pacing. This research would seem of interest to many scientists and perhaps also to the public. For example, although marathon training handbooks, magazines, and Web sites generally contain sound advice, their discussions of pacing might be improved considerably as the factors related to uneven pacing are identified.

Limitations. The current study is not without limitations. First, we evaluated pacing only by assessing first and second half split times, so we were unable to model when the slowing typically began. Other studies indicate that slowing becomes pronounced in the final $10-15 \mathrm{~km}$ of the marathon $(4,13,26)$. Second, we did not account for pacing variation associated with race courses or weather conditions and this variation may be substantial $(13,35)$. Third, although we succeeded in identifying experience factors associated with pacing variation, other experience factors that we did not assess may also be important. Similarly, as noted previously, we had no individual data on other relevant factors, including training, $\mathrm{CHO}$ ingestion, and targeted pace. Fourth, some runners may have participated in more than one race in our sample. We were unable to "cluster" performances under a given participant, and this could have led to CI values being smaller than they would have been if clustering was modeled explicitly.

\section{CONCLUSIONS}

This study demonstrates that the sex difference in pacing among nonelite marathon runners is robust and greater among slower runners. It occurs consistently across marathons, persists across age groups and ability levels, and remains when experience is controlled and when women's performances are adjusted to address men's greater $\mathrm{V}_{2 \max }$. The causes of the sex difference in pacing are presently unknown, but they likely involve the interplay of physiological and psychological factors. Studying this sex difference and related pacing phenomena should yield performance insights for scientists, coaches, and athletes.

This publication was partially supported by grant number UL1 TR000135 from the National Center for Advancing Translational Sciences. Its contents are solely the responsibility of the authors and do not necessarily represent the official views of the National Institutes of Health.

The authors thank Tyler Esser, Joseph Fleming, Alyssa Stevens, Jonathon Senefeld, and Marissa Miars for their assistance with data collection.

The authors declare no conflict of interest.

The results of the present study do not constitute endorsement by the American College of Sports Medicine.

\section{REFERENCES}

1. Abbiss CR, Laursen PB. Describing and understanding pacing strategies during athletic competition. Sports Med. 2008;38(3):239-52.

2. Allen EJ, Dechow PM. The "rationality" of the long distance runner: prospect theory and the marathon. Rochester (NY): Social Science Research Network; 2013[cited 2013 Dec 15]. Available from: http://papers.ssrn.com/abstract=2342396.

3. Brooks GA. Lactate: Link between glycolytic and oxidative metabolism. Sports Med. 2007;37(4-5):341-3.

4. Buman MP, Brewer BW, Cornelius AE, Van Raalte JL, Petitpas AJ. Hitting the wall in the marathon: phenomenological characteristics and associations with expectancy, gender, and running history. Psychol Sport Exerc. 2008;9(2):177-90.

5. Byrnes JP, Miller DC, Schafer WD. Gender differences in risk taking: a meta-analysis. Psychol Bull. 1999;125(3):367-83.

6. Callen KE. Mental and emotional aspects of long-distance running. Psychosomatics. 1983;24(2):133-51.
7. Carter SL, Rennie C, Tarnopolsky MA. Substrate utilization during endurance exercise in men and women after endurance training. Am J Physiol-Endocrinol Metab. 2001;280(6):E898-907.

8. Cheuvront SN, Carter R, DeRuisseau KC, Moffatt RJ. Running performance differences between men and women: an update. Sports Med. 2005;35(12):1017-24.

9. Coyle EF. Physiological regulation of marathon performance. Sports Med. 2007;37(4-5):306-11.

10. Croson R, Gneezy U. Gender differences in preferences. J Econ Lit. 2009;47(2):448-74.

11. Deaner RO. Distance running as an ideal domain for showing a sex difference in competitiveness. Arch Sex Behav. 2013;42(3): 413-28.

12. Dwyer PD, Gilkeson JH, List JA. Gender differences in revealed risk taking: evidence from mutual fund investors. Econ Lett. 2002;76(2):151-8. 
13. Ely MR, Martin DE, Cheuvront SN, Montain SJ. Effect of ambient temperature on marathon pacing is dependent on runner ability. Med Sci Sports Exerc. 2008;40(9):1675-80.

14. Gagnon D, Kenny GP. Does sex have an independent effect on thermoeffector responses during exercise in the heat? $J$ Physiol. 2012;590(23):5963-73.

15. Garratt RJ, Weinberger C, Johnson N. The State Street Mile: age and gender differences in competition aversion in the field. Econ Inq. 2013;51(1):806-15.

16. Gonzalez-Alonso J, Crandall CG, Johnson JA. The cardiovascular challenge of exercising in the heat. $J$ Physiol. 2008;586(1):45-53.

17. Halko M-L, Kaustia M, Alanko E. The gender effect in risky asset holdings. J Econ Behav Organ. 2012;83(1):66-81.

18. Harris CR, Jenkins M, Glaser D. Gender differences in risk assessment: why do women take fewer risks than men? Judgm Decis Making J. 2006;1(1):48-63.

19. Hunter SK. Sex differences in human fatigability: mechanisms and insight into physiological responses. Acta Physiol (Oxf). 2014; 10(4):768-89.

20. Hunter SK, Stevens AA. Sex differences in marathon running with advanced age: physiology or participation? Med Sci Sports Exerc. 2013;45(1):148-56.

21. Hunter SK, Stevens AA, Magennis K, Skelton KW, Fauth M. Is there a sex difference in the age of elite marathon runners? Med Sci Sports Exerc. 2011;43(4):656-64.

22. Johnsgard K. The motivation of the long-distance runner: 1. J Sports Med Phys Fitness. 1985;25(3):135-9.

23. Johnson JE, Powell PL. Decision making, risk and gender: are managers different? Br J Manag. 1994;5(2):123-8.

24. Joyner MJ. Physiological limiting factors and distance running: influence of gender and age on record performance. Exerc Sport Sci Rev. 1993;21:103-33.

25. Lamont LS. Gender differences in amino acid use during endurance exercise. Nutr Rev. 2005;63(12):419-22.
26. March DS, Vanderburgh PM, Titlebaum PJ, Hoops ML. Age, sex, and finish time as determinants of pacing in the marathon. $J$ Strength Cond Res. 2011;25(2):386-91.

27. Maughan RJ, Watson P, Shirreffs SM. Heat and cold: what does the environment do to the marathon runner? Sports Med. 2007; 37(4-5):396-9.

28. Muehlbauer T, Panzer S, Schindler C. Pacing pattern and speed skating performance in competitive long-distance events. $J$ Strength Cond Res. 2010;24(1):114-9.

29. Ogles BM, Masters KS. A typology of marathon runners based on cluster analysis of motivations. J Sport Behav. 2003;26(1):69-85.

30. Rapoport BI. Metabolic factors limiting performance in marathon runners. PLoS Comput Biol. 2010;6(10):e1000960, [cited 2013 Dec 18].

31. Roepstorff C, Steffensen CH, Madsen M, et al, Gender differences in substrate utilization during submaximal exercise in endurance-trained subjects. Am J Physiol-Endocrinol Metab. 2002;282(2):E435-47.

32. Sparling PB, O'Donnell EM, Snow TK. The gender difference in distance running performance has plateaued: an analysis of world rankings from 1980 to 1996. Med Sci Sports Exerc. 1998;30(12):1725-9.

33. Stevinson CD, Biddle SJ. Cognitive orientations in marathon running and "hitting the wall." Br J Sports Med. 1998;32(3):229-34.

34. Tarnopolsky MA. Sex differences in exercise metabolism and the role of 17-beta estradiol. Med Sci Sports Exerc. 2008;40(4):648-54.

35. Trubee NW. The effects of age, sex, heat stress, and finish time on pacing in the marathon [Internet]. 2011 [cited 2013 Jan 4]. Available from: http://etd.ohiolink.edu/view.cgi/Trubee $\% 20$ Nicholas.pdf? dayton 1303833182.

36. Venables MC, Achten J, Jeukendrup AE. Determinants of fat oxidation during exercise in healthy men and women: a crosssectional study. J Appl Physiol (1985). 2005;98(1):160-7.

37. Vleck VE, Bentley DJ, Millet GP, Burgi A. Pacing during an elite Olympic distance triathlon: comparison between male and female competitors. J Sci Med Sport. 2008;11(4):424-32. 